Louisiana State University

LSU Digital Commons

Faculty Publications

Department of Mathematics

$1-18-2018$

\title{
Sequential predictors under time-varying delays: Effects of delayed state observations in dynamic controller
}

Jerome Weston

Louisiana State University

Michael Malisoff

Louisiana State University

Frederic Mazenc

Laboratoire des Signaux et Systèmes

Follow this and additional works at: https://digitalcommons.Isu.edu/mathematics_pubs

\section{Recommended Citation}

Weston, J., Malisoff, M., \& Mazenc, F. (2018). Sequential predictors under time-varying delays: Effects of delayed state observations in dynamic controller. 2017 IEEE 56th Annual Conference on Decision and Control, CDC 2017, 2018-January, 4351-4356. https://doi.org/10.1109/CDC.2017.8264301

This Conference Proceeding is brought to you for free and open access by the Department of Mathematics at LSU Digital Commons. It has been accepted for inclusion in Faculty Publications by an authorized administrator of LSU Digital Commons. For more information, please contact ir@lsu.edu. 


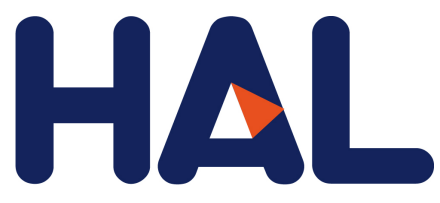

archives-ouvertes

\title{
Sequential Predictors under Time-Varying Delays: Effects of Delayed State Observations in Dynamic Controller
}

\author{
Jerome Weston, Michael Malisoff, Frédéric Mazenc
}

\section{To cite this version:}

Jerome Weston, Michael Malisoff, Frédéric Mazenc. Sequential Predictors under Time-Varying Delays: Effects of Delayed State Observations in Dynamic Controller. 56th IEEE Conference on Decision and Control (CDC 2017), Dec 2017, Melbourne, Australia. pp.4351-4356, 10.1109/cdc.2017.8264301. hal-01660132

\section{HAL Id: hal-01660132 \\ https://hal.inria.fr/hal-01660132}

Submitted on 10 Dec 2017

HAL is a multi-disciplinary open access archive for the deposit and dissemination of scientific research documents, whether they are published or not. The documents may come from teaching and research institutions in France or abroad, or from public or private research centers.
L'archive ouverte pluridisciplinaire HAL, est destinée au dépôt et à la diffusion de documents scientifiques de niveau recherche, publiés ou non, émanant des établissements d'enseignement et de recherche français ou étrangers, des laboratoires publics ou privés. 


\section{Sequential Predictors under Time-Varying Delays: Effects of Delayed State Observations in Dynamic Controller}

Jerome Weston
Michael Malisoff

Frederic Mazenc

\begin{abstract}
In a 2016 IEEE Conference on Decision and Control paper, our team designed sequential predictors for timevarying linear systems with time-varying delays, to prove global exponential stabilization properties using a feedback control that is computed in terms of the state of the last sequential predictor. This allowed feedback delays of arbitrarily large sup norm in the original system. Here we provide a significant generalization to more challenging cases with arbitrarily large feedback delay bounds, and where, in addition, current values of the plant state are not available to use in the sequential predictors. We illustrate our work in a pendulum example.
\end{abstract}

Index Terms-Delays, robustness, time-varying systems

\section{INTRODUCTION}

Sequential predictors [18] (which are also called chain predictors) provide an emerging technique to globally asymptotically stabilize systems under arbitrarily long feedback delays. The dynamic extensions in sequential prediction contain copies of the original system running at different time scales, with each extension also involving additional stabilizing terms. The sequential predictor approach is a recent development in a long history of research on stabilization under long input delays, which has its origins in the Smith predictor for linear systems [23], and work by Artstein [3], Krstic [10], and other important researchers that began during the 1980's and 1990s; see [8], [19], and [20] for more recent work on delay systems, and [24] and [25] for a valuable truncated predictor approach. Control design under input delays is motivated by many engineering applications. See, e.g., [4], [16], and [21] for overviews of delay compensating control, [22] for constant delays in neuromuscular electrical stimulation (or NMES), and [15] for analogs of NMES under time-varying delays.

Standard prediction involves replacing delayed state values in the feedback by predicted values, to eliminate feedback delays, but typically produces distributed terms that may not always be easy to compute [2] (but see, e.g., [1], [5], [6], and [26] for predictive controls that have no distributed terms for special cases of time invariant systems, and related results [7] on chain observers that do not cover the problems we address here). An advantage of existing sequential predictors is that they have no distributed terms, but previously published sequential predictor methods for time-varying linear systems

Malisoff and Weston are with the Department of Mathematics, Louisiana State University, Baton Rouge, LA 70803-4918, USA, malisoff@lsu.edu and jwesto3@lsu.edu.

Mazenc is with EPI DISCO INRIA-Saclay, Laboratoire des Signaux et Systèmes (L2S, UMR CNRS 8506), CNRS, CentraleSupélec, Université Paris-Sud, 3 rue Joliot Curie, 91192, Gif-sur-Yvette, France, Frederic.MAZENC@1ss.supelec.fr.

Malisoff and Weston were supported by NSF Grant 1408295
[12] require that the current state $x(t)$ of the original systems be available to use in the predictors when the control is computed. This can be a limitation when the sequential predictor must be computed on a computer that is far from the physical system, which can cause delays in the transmission of the state measurements from the physical system to the dynamic controller. This can occur for small robotic vehicles whose controls are computed remotely (as in our team's work [17] on marine robots) and is modeled by replacing $x(t)$ in the first sequential predictor by $x(t-\tau(t))$ with measurement delays $\tau$.

Therefore, this work studies the combined effects of (i) feedback delays $h(t)$ in the original system and (ii) measurement delays $\tau(t)$ in the sequential predictors. As in our team's paper [12], the new method in this work can allow arbitrarily long feedback delays $h(t)$ in the original system, but [12] did not allow delays $\tau(t)$ in the state values in the sequential predictors, and in addition, we illustrate below how our new method can lead to a smaller number of required sequential predictors than were required by [12]. Hence, this work can benefit engineering systems that contain measurement delays, and reduce the computational burden relative to [12].

\section{MAIN RESUlt}

We study systems of the form

$$
\dot{x}(t)=A(t) x(t)+B(t) u(t-h(t)),
$$

whose state $x$ and control $u$ are valued in $\mathbb{R}^{n}$ and $\mathbb{R}^{\ell}$, respectively, for any dimensions $n$ and $\ell$, where $h: \mathbb{R} \rightarrow$ $[0,+\infty)$ is a known time-varying delay. The dynamics (1) will be interconnected with a dynamic controller whose right side uses delayed values $x(t-\tau(t))$ of the state, where the delay $\tau: \mathbb{R} \rightarrow[0,+\infty)$ may differ from $h$. We make these assumptions, which agree with those of [12] when $\tau=0$ :

Assumption 1: The nonnegative valued functions $h$ and $\tau$ are $C^{1}$ and bounded from above by constants $c_{h}>0$ and $c_{\tau} \geq 0$ respectively, their first derivatives $\dot{h}$ and $\dot{\tau}$ have finite lower bounds, $\dot{h}$ and $\dot{\tau}$ are bounded from above by constants $l_{h} \in(0,1)$ and $l_{\tau} \in(0,1)$ respectively, and $\dot{h}$ has a global Lipschitz constant $n_{h}>0$.

Assumption 2: The functions $A$ and $B$ in (1) are bounded and continuous on $\mathbb{R}$, and there is a known bounded continuous function $K:[0,+\infty) \rightarrow \mathbb{R}^{\ell \times n}$ such that

$$
\dot{x}(t)=[A(t)+B(t) K(t)] x(t)
$$

is uniformly globally exponentially stable on $\mathbb{R}^{n}$ to 0 . 
We also assume throughout that the initial functions at time 0 are constant, e.g., $x(\ell)$ is constant on $(-\infty, 0]$. Assumption 1 can model many delays, e.g., using a denseness argument to closely approximate non- $C^{1}$ delays, including the approximation of discontinuous delays [12]. In terms of an integer $m>1$ that we specify later, we set

$$
\Omega_{i}(t)=t-\frac{i}{m} h(t) \text { and } \theta_{j}(t)=\Omega_{m-j+1}^{-1}\left(\Omega_{m-j}(t)\right)
$$

for all $i \in\{0, \ldots, m\}$ and $j \in\{1, \ldots, m\}$, and define

$$
R_{1}=\dot{\theta}_{1} \text { and } R_{i}(t)=\dot{\theta}_{i}(t) R_{i-1}\left(\theta_{i}(t)\right), 2 \leq i \leq m .
$$

The preceding functions were also used in [12], and are used to define the coefficients in our predictors, and they exist by our upper bounds $c_{h}$ and $l_{h} \in(0,1)$ from Assumption 1, which imply that the $\Omega_{i}$ 's are strictly increasing and that their ranges are all of $\mathbb{R}$, so the $\theta_{i}$ 's are also $C^{1}$ and strictly increasing. The inverses in (3) can be computed numerically using standard programs, e.g., Mathematica [11]. Setting

$$
u_{c}=n_{h} \frac{c_{h}}{\left(1-l_{h}\right)^{2}}+\frac{l_{h}}{1-l_{h}},
$$

we then prove the following, where $I_{n}$ is the $n \times n$ identity matrix, $|\cdot|$ is the usual Euclidean norm, and $|\cdot|_{\infty}\left(\right.$ resp., $\left.|\cdot|_{\mathcal{I}}\right)$ will denote the essential supremum over $[0,+\infty$ ) (resp., any interval $\mathcal{I} \subseteq[0,+\infty)$ ) in the Euclidean norm:

Theorem 1: If Assumptions 1-2 hold, and if the constant integer $m \geq 2$ satisfies

$$
2\left(1+\left(1+\frac{u_{c}}{m}\right)^{m}|A|_{\infty}\right)^{2} \sqrt{\frac{1+\frac{u_{c}}{m}}{1-l_{\tau}}}\left(c_{\tau}+\frac{c_{h}}{m\left(1-l_{h}\right)}\right)<1,
$$

then we can construct positive constants $\mu_{1}$ and $\mu_{2}$ such that for all solutions $x(t)$ of (1) in closed loop with the control

$$
u(t)=K\left(\Omega_{m}^{-1}(t)\right) z_{m}(t),
$$

where $z_{m}$ is the last $n$ components of the system

$$
\begin{aligned}
\dot{z}_{i}(t)= & R_{i}(t)\left(A\left(G_{i}(t)\right) z_{i}(t)+B\left(G_{i}(t)\right) u\left(\Omega_{m-i}(t)\right)\right) \\
& +L_{i}(t) \mathcal{E}_{i}\left(\theta_{i}^{-1}(\phi(t))\right), 1 \leq i \leq m
\end{aligned}
$$

having the state space $\mathbb{R}^{n m}$, and with the choices

$$
\begin{aligned}
& L_{i}(t)=-I_{n}-R_{i}(t) A\left(G_{i}(t)\right), \phi(t)=t-\tau(t), \\
& \text { and } G_{i}(t)=\Omega_{m}^{-1}\left(\Omega_{m-i}(t)\right),
\end{aligned}
$$

we have

$$
|(x(t), \mathcal{E}(t))| \leq \mu_{1}|(x, \mathcal{E})|_{\left[-c_{h}-c_{\tau}, 0\right]} e^{-\mu_{2} t}
$$

for all $t \geq 0$, where $\mathcal{E}(t)=\left(z_{1}(t)-x\left(\theta_{1}(t)\right), z_{2}(t)-\right.$ $\left.z_{1}\left(\theta_{2}(t)\right), \ldots, z_{m}(t)-z_{m-1}\left(\theta_{m}(t)\right)\right)$.

Before proving Theorem 1, we provide several remarks on the novelty and value of the theorem.

\section{Remarks On Novelty And VAlue of TheOrem 1}

Remark 1: The $z_{i}$ dynamics in (8) is called the $i$ th sequential (sub)predictor for $i=1,2, \ldots, m$ and has state space $\mathbb{R}^{n}$, so (7) only depends on time and on the state of the last sequential predictor. Our system (8) has the advantage that it does not depend on current $x(t)$ values (because of the $\phi$ entering the $\mathcal{E}_{1}\left(\theta_{1}^{-1}(\phi(t))\right)$ in the first sequential predictor in
(8)), so the current state $x(t)$ is not needed when computing the $z_{i}$ 's at times $t$. Our system (8) also involves delays $\tau$ through the $\mathcal{E}_{i}\left(\theta_{i}^{-1}(\phi(t))\right)$ 's for $i \geq 2$, since these delays are also needed for our global exponential stability proof. When $\tau=0$, we can satisfy (6) by choosing $m$ large enough, so we allow arbitrarily large bounds $c_{h}$ on the delay $h$.

However, since there are no sequential predictors available to compensate for the delays $\tau(t)$ in (8), condition (6) is needed to put a restriction on the allowable $c_{\tau}$ 's. By using the definition of $e$ as a limit to obtain

$$
e^{u_{c}}=\lim _{m \rightarrow \infty}\left(\left(1+\frac{u_{c}}{m}\right)^{m / u_{c}}\right)^{u_{c}}
$$

it follows that (6) holds if $m$ is large enough and if

$$
\frac{c_{\tau}}{\sqrt{1-l_{\tau}}} \in\left[0, \frac{1}{2\left(1+e^{u c}|A|_{\infty}\right)^{2}}\right) \text {. }
$$

Condition (12) is not too restrictive, since the $\tau$ 's from (8) only arise from measurement delays, whereas the delay $h(t)$ in (1) comes from both transmission delays from the $m$ th sequential predictor to the physical plant, and from delays in the control actuation in (1). Hence, in practice, the feedback delay $h$ would be much larger than $\tau$ in the sup norm.

Remark 2: Theorem 1 is notable even in the special case where $\tau(t)$ is the zero function, since it can allow a smaller number $m$ of sequential predictors than were allowed in [12]. The work [12] required the delay $\tau$ in the $x$ observations in the dynamic control (8) to be the zero function and that the number $m$ of sequential predictors satisfy

$$
\max \left\{2,4\left(\frac{b_{1}}{\sqrt{2}}+b_{3}\right) \frac{c_{h}}{1-l_{h}}\right\}<m,
$$

where, in terms of our notation from (5) and Assumption 1,

$b_{1}=\left[1+\left(1+\frac{u_{c}}{m}\right)^{m}|A|_{\infty}\right]\left(1+\frac{u_{c}}{m}\right)^{m}|A|_{\infty}$,

$b_{2}=\left[1+\left(1+\frac{u_{c}}{m}\right)^{m}|A|_{\infty}\right]^{2}$, and $b_{3}=b_{2}\left(1+\frac{u_{c}}{m}\right)$.

In Section VI, we illustrate how (6) can lead to a significantly smaller number of required sequential predictors as compared with (13) when $\tau$ is the zero function. Our example also illustrates how we can allow nonzero $\tau$ 's.

Remark 3: The estimate (10) implies that the $(x, \mathcal{E})$ dynamics are uniformly globally exponentially stable to 0 . Key features of Theorem 1 are (a) our allowing arbitrarily large bounds $c_{h}$ on $h$ as well as measurement delays $\tau$, (b) that our control (7) has no distributed terms, and (c) that only the bottom $n$ components of (8) are needed to compute (7).

\section{Three Lemmas to Prove Theorem 1}

Our proof of Theorem 1 will follow from three lemmas. The first lemma was shown in [12], and the second follows from simple calculations as were done in Part 1 of the proof of [12, Theorem 1] except with $\mathcal{E}_{i}\left(\theta_{i}^{-1}(t)\right)$ in the dynamics for the error variable $\mathcal{E}$ in [12] replaced by $\mathcal{E}_{i}\left(\theta_{i}^{-1}(\phi(t))\right)$ for each $i$, so we only prove our third lemma in this section.

Lemma 1: The $\theta_{i}$ 's in (3) and the constant (5) satisfy

$$
\left|\dot{\theta}_{i}(\ell)-1\right| \leq \frac{u_{c}}{m} \text { and }\left|\theta_{i}^{-1}(\ell)-\ell\right| \leq \frac{c_{h}}{1-l_{h}} \frac{1}{m}
$$

for all $\ell \in \mathbb{R}$ and $i \in\{1,2, \ldots, m\}$. 
Lemma 2: The dynamics for the variable $\mathcal{E}$ in Theorem 1 are

$$
\left\{\begin{aligned}
\dot{\mathcal{E}}_{1}(t)= & R_{1}(t) A\left(G_{1}(t)\right) \mathcal{E}_{1}(t) \\
& +L_{1}(t) \mathcal{E}_{1}\left(\theta_{1}^{-1}(\phi(t))\right) \\
\dot{\mathcal{E}}_{i}(t)= & R_{i}(t) A\left(G_{i}(t)\right) \mathcal{E}_{i}(t)+L_{i}(t) \mathcal{E}_{i}\left(\theta_{i}^{-1}(\phi(t))\right) \\
& -\dot{\theta}_{i}(t) L_{i-1}\left(\theta_{i}(t)\right) \\
& \times \mathcal{E}_{i-1}\left(\theta_{i-1}^{-1}\left(\phi\left(\theta_{i}(t)\right)\right)\right), 2 \leq i \leq m
\end{aligned}\right.
$$

Also, the system (1) in closed loop with (7)-(8) can be written as

$$
\begin{aligned}
& \dot{x}(t)=(A(t)+B(t) K(t)) x(t) \\
& +\left\{B ( t ) \left[K(t) \mathcal{E}_{1}\left(\theta_{2}\left(\ldots \theta_{m}(t-h(t) \ldots)\right)\right)\right.\right. \\
& \left.\left.+\ldots+K(t) \mathcal{E}_{m}(t-h(t))\right]\right\}
\end{aligned}
$$

and

$$
z_{m}(t)=x\left(\Omega_{m}^{-1}(t)\right)+\mathcal{E}_{1}\left(\theta_{2}\left(\ldots \theta_{m}(t) \ldots\right)\right)+\ldots+\mathcal{E}_{m}(t)
$$

holds for all $t \geq 0$. Finally, $\theta_{i}^{-1}(t) \leq t$ holds for all $t \geq 0$ and all $i \in\{1,2, \ldots, m\}$.

Setting

$$
c_{m}^{\sharp}=c_{\tau}+\frac{c_{h}}{m\left(1-l_{h}\right)},
$$

and $f_{t}(s)=f(t+s)$ for any function $f$ and $t \geq 0$ and $s \leq 0$ for which the equality holds, our final lemma is:

Lemma 3: Let the assumptions of Theorem 1 hold, $i \in$ $\{1,2, \ldots, m\}$ be given, and $\lambda>1$ be a constant such that $\lambda \mathcal{H}<1$, where $\mathcal{H}$ denotes the left side of (6). Then there is a constant $c_{0}>0$ such that the time derivative of

$$
\begin{aligned}
& Q^{\sharp}\left(s_{t}\right)= \\
& \frac{1}{2}|s(t)|^{2}+\left(1+\left|R_{i}\right|_{\infty}|A|_{\infty}\right)^{4} \frac{\lambda^{2} c_{m}^{\sharp}}{1-l_{\tau}}\left(1+\frac{u_{c}}{m}\right) \mathcal{I}\left(s_{t}\right),
\end{aligned}
$$$$
\text { where } \mathcal{I}\left(s_{t}\right)=\int_{t-2 c_{m}^{\sharp}}^{t} \int_{\ell}^{t}|s(r)|^{2} \mathrm{~d} r \mathrm{~d} \ell
$$

along all solutions of

$$
\dot{s}(t)=R_{i}(t) A\left(G_{i}(t)\right) s(t)+L_{i}(t) s\left(\theta_{i}^{-1}(\phi(t))\right)
$$

satisfies

$$
\dot{Q}^{\sharp}(t) \leq-c_{0} Q^{\sharp}\left(s_{t}\right)
$$

for all $t \geq 0$.

Proof: Since $\theta_{i}^{-1}$ is strictly increasing, as a composition of the strictly increasing functions $\Omega_{m-i}^{-1}$ and $\Omega_{m-i+1}$, it follows from Lemma 2 that $\theta_{i}^{-1}(\phi(t)) \leq \theta_{i}^{-1}(t) \leq t$ for all $t \geq 0$. Also, our choices of the $L_{i}$ 's in (9) and the Fundamental Theorem of Calculus let us rewrite (21) as

$$
\dot{s}(t)=-s(t)+\left[I_{n}+R_{i}(t) A\left(G_{i}(t)\right)\right] \mathcal{D}_{i}(t),
$$

where $\mathcal{D}_{i}(t)=s(t)-s\left(\theta_{i}^{-1}(\phi(t))\right)$, and where for all $t \geq 0$,

$$
\begin{aligned}
\left|\mathcal{D}_{i}(t)\right| \leq & \int_{\theta_{i}^{-1}(\phi(t))}^{t}\left|R_{i}(\ell) A\left(G_{i}(\ell)\right) s(\ell)\right| \mathrm{d} \ell \\
& +\int_{\theta_{i}^{-1}(\phi(t))}^{t}\left|L_{i}(\ell) s\left(\theta_{i}^{-1}(\phi(\ell))\right)\right| \mathrm{d} \ell,
\end{aligned}
$$

by the constantness of the initial functions at time 0 .

Then the second inequality in (15) in Lemma 1 (applied with $\ell=\phi(t)$ ) gives

$$
\begin{aligned}
& 0 \leq t-\theta_{i}^{-1}(\phi(t))=\tau(t)+\phi(t)-\theta_{i}^{-1}(\phi(t)) \\
& \leq \tau(t)+\left|\phi(t)-\theta_{i}^{-1}(\phi(t))\right| \leq \tau(t)+\frac{c_{h}}{m\left(1-l_{h}\right)} \leq c_{m}^{\sharp}
\end{aligned}
$$

for all $t \geq 0$. Therefore, for all $t \geq 0$, we can now use Jensen's inequality (applied to the convex squaring function) to obtain

$$
\left(\int_{\theta_{i}^{-1}(\phi(t))}^{t}|s(\ell)| \mathrm{d} \ell\right)^{2} \leq c_{m}^{\sharp} \int_{\theta_{i}^{-1}(\phi(t))}^{t}|s(\ell)|^{2} \mathrm{~d} \ell .
$$

Also, (15) and (25) and the change of variables $u=$ $\theta_{i}^{-1}(\phi(\ell))$ give

$$
\begin{aligned}
& \left(\int_{\theta_{i}^{-1}(\phi(t))}^{t}\left|s\left(\theta_{i}^{-1}(\phi(\ell))\right)\right| \mathrm{d} \ell\right)^{2} \\
& \leq\left(\int_{t-\tau(t)-\frac{c_{h}}{m\left(1-l_{h}\right)}}^{t}\left|s\left(\theta_{i}^{-1}(\phi(\ell))\right)\right| \mathrm{d} \ell\right)^{2} \\
& \leq c_{m}^{\sharp} \int_{\phi(t)-\frac{c_{h}}{m\left(1-l_{h}\right)}}^{t}\left|s\left(\theta_{i}^{-1}(\phi(\ell))\right)\right|^{2} \mathrm{~d} \ell \\
& \leq c_{m}^{\sharp} \int_{\theta_{i}^{-1}\left(\phi(t)-c_{m}^{\sharp}\right)}^{\theta_{i}^{-1}(\phi(t))}|s(u)|^{2} \frac{\dot{\theta}_{i}(u)}{1-l_{\tau}} \mathrm{d} u,
\end{aligned}
$$

where the last inequality followed by combining the bound $\phi\left(\phi(t)-\left(c_{h} /\left(m\left(1-l_{h}\right)\right)\right)\right) \geq \phi(t)-c_{m}^{\sharp}$ with the fact that $\theta_{i}^{-1}$ is strictly increasing and from the bounds $\dot{\tau}(t) \leq l_{\tau}<1$.

We next combine the estimates (26)-(27) with (23) to get a suitable decay estimate on $Q(t)=|s(t)|^{2} / 2$ along solutions of (23), as follows. We first introduce the functions

$$
\begin{aligned}
\mathcal{P}_{i}(t)= & \left(1+\left|R_{i}\right|_{\infty}|A|_{\infty}\right) \times \\
& \int_{\theta_{i}^{-1}(\phi(t))}^{t}\left|R_{i}(\ell)\right|\left|A\left(G_{i}(\ell)\right)\right||s(\ell)| \mathrm{d} \ell
\end{aligned}
$$

and

$$
\begin{aligned}
\mathcal{J}_{i}(t)= & \left(1+\left|R_{i}\right|_{\infty}|A|_{\infty}\right) \times \\
& \int_{\theta_{i}^{-1}(\phi(t))}^{t}\left|L_{i}(\ell)\right|\left|s\left(\theta_{i}^{-1}(\phi(\ell))\right)\right| \mathrm{d} \ell .
\end{aligned}
$$

We next use the facts that $\left|L_{i}\right|_{\infty} \leq 1+\left|R_{i}\right|_{\infty}|A|_{\infty}$ and $\left|\dot{\theta}_{i}(t)\right| \leq 1+u_{c} / m$ both hold for all $t \geq 0$ (by the first inequality in Lemma 1) and Young's Inequality $a b \leq$ $0.25 a^{2}+b^{2}$ twice (with the choices $a=|s(t)|$, and with $b=\mathcal{P}_{i}(t)$ and then with $\left.b=\mathcal{J}_{i}(t)\right)$ to obtain

$$
\begin{aligned}
\dot{Q}(t) \leq & -|s(t)|^{2}+|s(t)| \mathcal{P}_{i}(t)+|s(t)| \mathcal{J}_{i}(t) \\
\leq & -\frac{1}{2}|s(t)|^{2}+\left[\alpha_{i 1} \int_{\theta_{i}^{-1}(\phi(t))}^{t}|s(\ell)|^{2} \mathrm{~d} \ell\right. \\
& \left.+\alpha_{i 2} \int_{t-2 c_{m}^{\sharp}}^{\theta_{i}^{-1}(\phi(t))}|s(u)|^{2} \mathrm{~d} u\right]
\end{aligned}
$$

along all solutions of (23), where

$$
\begin{aligned}
\alpha_{i 1} & =\left(1+\left|R_{i}\right|_{\infty}|A|_{\infty}\right)^{2}\left|R_{i}\right|_{\infty}^{2}|A|_{\infty}^{2} c_{m}^{\sharp} \text { and } \\
\alpha_{i 2} & =\left(1+\left|R_{i}\right|_{\infty}|A|_{\infty}\right)^{4} \frac{c_{m}^{\sharp}}{1-l_{\tau}}\left(1+\frac{u_{c}}{m}\right),
\end{aligned}
$$

and where we also used (15) and (24)-(25) and

$$
\theta_{i}^{-1}\left(\phi(t)-c_{m}^{\sharp}\right) \geq \theta_{i}^{-1}\left(t-c_{m}^{\sharp}-c_{\tau}\right) \geq t-2 c_{m}^{\sharp}
$$

and (26)-(27) to upper bound $\mathcal{P}_{i}^{2}$ and $\mathcal{J}_{i}^{2}$, respectively.

We next use the facts that for all $t \geq 0$, we have

$$
\frac{d}{d t} \mathcal{I}\left(s_{t}\right)=2 c_{m}^{\sharp}|s(t)|^{2}-\int_{t-2 c_{m}^{\sharp}}^{t}|s(r)|^{2} \mathrm{~d} r
$$

and

$$
\mathcal{I}\left(s_{t}\right) \leq 2 c_{m}^{\sharp} \int_{t-2 c_{m}^{\sharp}}^{t}|s(r)|^{2} \mathrm{~d} r .
$$

Since $\alpha_{i 1} \leq \alpha_{i 2}$, and since (20) can be written as $Q^{\sharp}\left(s_{t}\right)=$ 
$\frac{1}{2}|s(t)|^{2}+\alpha_{i 2} \lambda^{2} \mathcal{I}\left(s_{t}\right)$, it follows from combining the two integrals in (30) and then using (33) that the time derivative of $Q^{\sharp}$ along all solutions of (23) satisfies

$$
\begin{aligned}
& \dot{Q}^{\sharp}(t) \leq\left[-\frac{1}{2}+2 \lambda^{2} c_{m}^{\sharp} \alpha_{i 2}\right]|s(t)|^{2}+\left(1-\lambda^{2}\right) W_{i}(t) \\
& \text { where } W_{i}(t)=\alpha_{i 2} \int_{t-2 c_{m}^{\sharp}}^{t}|s(\ell)|^{2} \mathrm{~d} \ell .
\end{aligned}
$$

By (6) and our choice of $\lambda>1$ and the fact that (15) gives $\left|R_{i}(t)\right| \leq\left(1+\left(u_{c} / m\right)\right)^{m}$ for all $t \geq 0$, we conclude that the coefficients of $|s(t)|^{2}$ and $W_{i}(t)$ on the right side of (35) are negative. Hence, we can use (34) to check that

$$
c_{0}=\min \left\{1-4 \lambda^{2} c_{m}^{\sharp} \alpha_{i 2}, \frac{\lambda^{2}-1}{2 \lambda^{2} c_{m}^{\sharp}}\right\}
$$

satisfies our requirements. This proves Lemma 3.

\section{Proof of Theorem 1}

We first study the dynamics for the transformed error vector $\mathcal{E}^{\sharp}(t)=\left(\mathcal{E}_{1}\left(\kappa_{1}(t)\right), \ldots, \mathcal{E}_{m}\left(\kappa_{m}(t)\right)\right)$ on the set $\{t \geq$ $\left.0: \min \left\{\kappa_{i}(t): 1 \leq i \leq m\right\} \geq 0\right\}$, where $\kappa_{1}(t)=t$ and $\kappa_{i+1}=\theta_{i+1}^{-1} \circ \phi^{-1} \circ \theta_{i} \circ \kappa_{i}$ for $i=1, \ldots, m$, and where $\circ$ means composition. Then

$$
\theta_{i}^{-1} \circ \phi \circ \theta_{i+1} \circ \kappa_{i+1}=\kappa_{i} \text { for } i=1,2, \ldots, m .
$$

Note that Assumption 1 ensures that there are positive constants $\underline{\theta}$ and $\bar{\theta}$ such that $\underline{\theta} \leq \kappa_{i}^{\prime}(t) \leq \bar{\theta}$ hold for all $i \in\{1,2, \ldots, m\}$ and $t \in \mathbb{R}$ (by noting that there are positive constants $\underline{a}$ and $\bar{a}$ such that for all $t \in \mathbb{R}$, we have $\underline{a} \leq \Omega_{i}^{\prime}(t) \leq \bar{a}$ and $\underline{a} \leq\left(\Omega_{i}^{-1}\right)^{\prime}(t) \leq \bar{a}$ for all $i$ and $\underline{a} \leq\left(\phi^{-1}\right)^{\prime}(t) \leq \bar{a}$, and then reapplying the chain rule).

The decay estimate (22) on $Q^{\sharp}$, combined with Young's inequality $a b \leq \frac{1}{4} a^{2}+b^{2}$ for suitable $a \geq 0$ and $b \geq 0$ and our choice of the quadratic part of $Q^{\sharp}$, implies that for all $t \geq 0$ in the set $\mathcal{S}$ of all $t \geq 0$ such that $\min \left\{\kappa_{i}(t): 1 \leq\right.$ $i \leq m\} \geq 0$ and along all solutions of the dynamics for $\left(\mathcal{E}_{1}^{\sharp}(t), \mathcal{E}_{2}^{\sharp}(t)\right)=\left(\mathcal{E}_{1}\left(\kappa_{1}(t)\right), \mathcal{E}_{2}\left(\kappa_{2}(t)\right)\right)$, we have

$$
\begin{aligned}
\frac{d}{d t} Q^{\sharp}\left(\mathcal{E}_{2, t}^{\sharp}\right) \leq & -\frac{\underline{\theta} c_{0}}{2} Q^{\sharp}\left(\mathcal{E}_{2, t}^{\sharp}\right) \\
& +\left\{\frac{2 \bar{\theta}^{4}}{\underline{\theta} c_{0}}\left(\left|\dot{\theta}_{2}\right|_{\infty}\left|L_{1}\right|_{\infty}\right)^{2}\right\} Q^{\sharp}\left(\mathcal{E}_{1, t}^{\sharp}\right) \\
\frac{d}{d t} Q^{\sharp}\left(\mathcal{E}_{1, t}^{\sharp}\right) \leq & -\underline{\theta} c_{0} Q^{\sharp}\left(\mathcal{E}_{1, t}^{\sharp}\right),
\end{aligned}
$$

where the first inequality used (22), then Young's inequality, and then the fact that $\theta_{1}^{-1} \circ \phi \circ \theta_{2} \circ \kappa_{2}=\kappa_{1}$ to get

$$
\begin{aligned}
& \bar{\theta}^{2}\left|\mathcal{E}_{2}\left(\kappa_{2}(t)\right)\right|\left|\dot{\theta}_{2}\right|_{\infty}\left|L_{1}\right|_{\infty}\left|\mathcal{E}_{1}\left(\theta_{1}^{-1} \circ \phi \circ \theta_{2} \circ \kappa_{2}(t)\right)\right| \\
& \leq \frac{\underline{\underline{\theta}} c_{0}}{\underline{4}}\left|\mathcal{E}_{2}\left(\kappa_{2}(t)\right)\right|^{2} \\
& \quad+\frac{\bar{\theta}^{4}}{\underline{\theta} c_{0}}\left(\left|\dot{\theta}_{2}\right|_{\infty}\left|L_{1}\right|_{\infty}\right)^{2}\left|\mathcal{E}_{1}\left(\theta_{1}^{-1} \circ \phi \circ \theta_{2} \circ \kappa_{2}(t)\right)\right|^{2} \\
& \leq \frac{\underline{\theta} c_{0}}{2} Q^{\sharp}\left(\mathcal{E}_{2, t}^{\sharp}\right)+\frac{2 \bar{\theta}^{4}}{\underline{\theta} c_{0}}\left(\left|\dot{\theta}_{2}\right|_{\infty}\left|L_{1}\right|_{\infty}\right)^{2} Q^{\sharp}\left(\mathcal{E}_{1, t}^{\sharp}\right),
\end{aligned}
$$

by (16), where $\mathcal{E}_{i, t}(s)=\mathcal{E}_{i}(t+s)$ for all $s \leq 0$. Hence,

$Q_{2}^{\sharp}\left(\mathcal{E}_{1, t}^{\sharp}, \mathcal{E}_{2, t}^{\sharp}\right)=$

$Q^{\sharp}\left(\mathcal{E}_{2, t}^{\sharp}\right)+\left(1+\frac{1}{\underline{\theta} c_{0}}\left\{\frac{2 \bar{\theta}^{4}}{\underline{\theta} c_{0}}\left(\left|\dot{\theta}_{2}\right|_{\infty}\left|L_{1}\right|_{\infty}\right)^{2}\right\}\right) Q^{\sharp}\left(\mathcal{E}_{1, t}^{\sharp}\right)$

satisfies

$$
\dot{Q}_{2}^{\sharp}(t) \leq-\frac{\theta}{\frac{\theta}{c} c_{0}}\left(Q^{\sharp}\left(\mathcal{E}_{1, t}^{\sharp}\right)+Q^{\sharp}\left(\mathcal{E}_{2, t}^{\sharp}\right)\right)
$$

for all $t \in \mathcal{S}$ and so is a Lyapunov-Krasovskii functional for the $\left(\mathcal{E}_{1}^{\sharp}(t), \mathcal{E}_{2}^{\sharp}(t)\right)$ dynamics; we used the quantity in curly braces in (39) to cancel the effects of the nonnegative term in $(d / d t) Q^{\sharp}\left(\mathcal{E}_{2, t}^{\sharp}\right)$ in (38). Arguing inductively (by adding one component of $\mathcal{E}^{\sharp}$ at a time, and using (37), to cancel the effects of the nonnegative terms) provides positive constants $\omega_{i}$ and $\eta_{1}$ such that

$$
Q_{m}^{\sharp}\left(\mathcal{E}_{t}^{\sharp}\right)=\sum_{i=1}^{m} \omega_{i} Q^{\sharp}\left(\mathcal{E}_{i, t}^{\sharp}\right)
$$

satisfies

$$
\dot{Q}_{m}^{\sharp}\left(\mathcal{E}_{t}^{\sharp}\right) \leq-\eta_{1} Q_{m}^{\sharp}\left(\mathcal{E}_{t}^{\sharp}\right)
$$

along all solutions of the $\mathcal{E}^{\sharp}$ dynamics for all $t \in \mathcal{S}$.

Assumption 2 provides a $C^{1}$ bounded function $P$ such that $V(t, x)=x^{\top} P(t) x$ has a quadratic lower bound in $|x|$ of the form $p_{0}|x|^{2}$ for some constant $p_{0}>0$ and satisfies $\dot{V} \leq-|x(t)|^{2}$ along solutions of (2) (using [9, Theorem 4.14]). Then along all solutions of (17), we have $V(t, x(t)) \leq$ $|P|_{\infty}|x(t)|^{2}$, so the triangle inequality $a b \leq \frac{1}{2}\left(a^{2}+b^{2}\right)$ gives

$$
\begin{aligned}
\dot{V} \leq & -|x(t)|^{2}+\{|x(t)|\}\left\{2|P|_{\infty}\left|\delta^{\sharp}\right|_{\infty}\right\} \\
\leq \quad & -\frac{1}{2}|x(t)|^{2}+2|P|_{\infty}^{2}\left|\delta^{\sharp}\right|_{\infty}^{2} \\
\leq \quad & -\frac{1}{2|P|_{\infty}} V(t, x(t)) \\
& +2 m\left(|P|_{\infty}|B|_{\infty}|K|_{\infty}\right)^{2} \\
& \times\left(\left|\mathcal{E}_{1}\left(\theta_{2}\left(\ldots \theta_{m}(t-h(t) \ldots)\right)\right)\right|^{2}+\ldots\right. \\
& \left.+\left|\mathcal{E}_{m}(t-h(t))\right|^{2}\right)
\end{aligned}
$$

where $\delta^{\sharp}$ is the quantity in curly braces in (17), by applying the triangle to the terms in curly braces in (43), and using the Cauchy inequality for squaring a sum of $m$ nonnegative terms to produce the $2 m$ factor in (43).

Set $\mathcal{M}_{i}=\kappa_{i}^{-1} \circ \theta_{i+1} \circ \cdots \circ \theta_{m} \circ \Omega_{m}$ if $1 \leq i \leq m-1$ and $\mathcal{M}_{m}=\kappa_{m}^{-1} \circ \Omega_{m}$. Then the same reasoning we used to find $\underline{\theta}$ and $\bar{\theta}$ provides positive constants $\underline{\mathcal{M}}$ and $\overline{\mathcal{M}}$ such that $\underline{\mathcal{M}} \leq \mathcal{M}_{i}^{\prime}(t) \leq \overline{\mathcal{M}}$ for all $t \in \mathbb{R}$ and $i$. Moreover,

$$
\begin{aligned}
& \left|\mathcal{E}_{i}\left(\theta_{i+1} \circ \ldots \circ \theta_{m}(t-h(t))\right)\right|^{2} \\
& =2 Q\left(\mathcal{E}_{i}\left(\theta_{i+1} \circ \ldots \circ \theta_{m} \circ \Omega_{m}(t)\right)\right) \\
& =2 Q\left(\mathcal{E}_{i}^{\sharp}\left(\mathcal{M}_{i}(t)\right)\right) \leq \frac{2}{\omega_{i}} Q_{m}^{\sharp}\left(\mathcal{E}_{\mathcal{M}_{i}(t)}^{\sharp}\right)
\end{aligned}
$$

for all $i \in\{1,2, \ldots, m-1\}$ (by (41)), and

$$
\left|\mathcal{E}_{m}(t-h(t))\right|^{2}=2 Q\left(\mathcal{E}_{m}\left(\Omega_{m}(t)\right)\right) \leq \frac{2}{\omega_{m}} Q_{m}^{\sharp}\left(\mathcal{E}_{\mathcal{M}_{m}(t)}^{\sharp}\right) .
$$

By the preceding upper bounds and (43), the function

$$
\begin{aligned}
& Q_{+}^{\sharp}\left(\mathcal{E}_{t}^{\sharp \sharp}\right)=V(t, x(t)) \\
& +\left(1+\frac{2 m\left(|P|_{\infty}|B|_{\infty}|K|_{\infty}\right)^{2}}{\underline{\mathcal{M}} \eta_{1}}\right) \sum_{i=1}^{m} \frac{2}{\omega_{i}} Q_{m}^{\sharp}\left(\mathcal{E}_{\mathcal{M}_{i}(t)}^{\sharp}\right)
\end{aligned}
$$

admits a constant $d_{0}>0$ such that

$$
\dot{Q}_{+}^{\sharp}\left(\mathcal{E}_{t}^{\sharp \sharp}\right) \leq-d_{0} Q_{+}^{\sharp}\left(\mathcal{E}_{t}^{\sharp \sharp}\right)
$$

holds along all solutions of the dynamics for

$$
\mathcal{E}^{\sharp \sharp}(t)=\left(x(t), \mathcal{E}^{\sharp}\left(\mathcal{M}_{1}(t)\right), \ldots, \mathcal{E}^{\sharp}\left(\mathcal{M}_{m}(t)\right)\right)
$$

for all $t \geq 0$ such that

$$
\min \left\{\min \left\{\kappa_{i}(t), \kappa_{i} \circ \mathcal{M}_{i}(t)\right\}: 1 \leq i \leq m\right\} \geq 0 .
$$


Since the $\kappa_{i}$ 's and $\mathcal{M}_{i}$ 's are strictly increasing and unbounded, we can find a constant $a_{*}>0$ such that (48) holds for all $t \geq a_{*}$. It follows from (46) that there are positive constants $c_{1}$ and $c_{2}$ such that

$$
\begin{aligned}
& \left|\mathcal{E}_{i}\left(\kappa_{i} \circ \mathcal{M}_{i}(t)\right)\right|^{2} \\
& \leq Q_{+}^{\sharp}\left(\mathcal{E}_{t}^{\sharp \sharp}\right) \leq c_{1}\left|\left(x, \mathcal{E}^{\sharp \sharp}\right)\right|_{\left[a_{*}-2 c_{m}^{\sharp}, a_{*}\right]}^{2} e^{-c_{2} t}, \quad 1 \leq i \leq m
\end{aligned}
$$

holds along all solutions of the dynamics for (47) for all $t \geq$ $a_{*}$. Since the $\kappa_{i} \circ \mathcal{M}_{i}$ 's are strictly increasing and unbounded, we can then find a constant $\bar{s}>a_{*}$ such that

$$
\begin{aligned}
& \left|\mathcal{E}_{i}(t)\right|^{2} \leq c_{1}\left|\left(x, \mathcal{E}^{\sharp \sharp}\right)\right|_{\left[a_{*}-2 c_{m}^{\sharp}, a_{*}\right]}^{2} e^{-c_{2}\left(\kappa_{i} \circ \mathcal{M}_{i}\right)^{-1}(t)} \\
& \text { and }|x(t)|^{2} \leq \frac{c_{1}}{p_{0}}\left|\left(x, \mathcal{E}^{\sharp \sharp}\right)\right|_{\left[a_{*}-2 c_{m}^{\sharp}, a_{*}\right]}^{2} e^{-c_{2} t}
\end{aligned}
$$

hold for all $t \geq \bar{s}$, e.g., any $\bar{s}>a_{*}$ such that $\min \left\{\kappa_{i}(t), \kappa_{i}\right.$ 。 $\left.\mathcal{M}_{i}(t),\left(\kappa_{i} \circ \mathcal{M}_{i}\right)^{-1}(t)\right\} \geq 0$ for all $i \in\{1,2, \ldots, m\}$ and $t \geq \bar{s}$; such a $\bar{s}$ exists because $\lim _{t \rightarrow+\infty} \theta_{i}(t)=$ $\lim _{t \rightarrow+\infty} \theta_{i}^{-1}(t)=\lim _{t \rightarrow+\infty} \Omega_{i}(t)=\lim _{t \rightarrow+\infty} \Omega_{i}^{-1}(t)=$ $+\infty$ for all $i \in\{1,2, \ldots, m\}$. Since we can find positive constants $h_{o}$ and $h_{1}$ such that $\left(\kappa_{i} \circ \mathcal{M}_{i}\right)^{-1}(t) \geq h_{o} t-h_{1}$ for all $i$ and all $t \in \mathbb{R}$ (by finding a positive lower bound on $\left(\left(\kappa_{i} \circ \mathcal{M}_{i}\right)^{-1}\right)^{\prime}(t)$ over all $i$ and $t$ and then applying the Mean Value Theorem to the $\left(\kappa_{i} \circ \mathcal{M}_{i}\right)^{-1}$ 's), it follows from (49) and the subadditivity of the square root that we can find positive constants $\omega_{1}$ and $\omega_{2}$ such that

$$
|(x(t), \mathcal{E}(t))| \leq \omega_{1}\left|\left(x, \mathcal{E}^{\sharp \sharp}\right)\right|_{\left[a_{*}-2 c_{m}^{\sharp}, a_{*}\right]} e^{-\omega_{2} t}
$$

holds along all solutions of the $(x, \mathcal{E})$ systems for all $t \geq \bar{s}$.

Next note that the formula for the $\mathcal{E}_{1}$ subsystem of (16) and the bounds $\theta_{i}^{-1}(\phi(t)) \leq \phi(t) \leq t$ provide a constant $c_{a}>0$ such that $\left|\dot{\mathcal{E}}_{1}(\ell)\right| \leq c_{a}\left|\mathcal{E}_{1}\right|_{\left[-c_{h}-c_{\tau}, \ell\right]}$ and so also

$$
\left|\mathcal{E}_{1}(\ell)\right| \leq\left|\mathcal{E}_{1}\right|_{\left[-c_{h}-c_{\tau}, 0\right]}+c_{a} \int_{0}^{\ell}\left|\mathcal{E}_{1}\right|_{\left[-c_{h}-c_{\tau}, s\right]} \mathrm{d} s
$$

for all $\ell \geq 0$. For all $t \geq 0$, this gives

$$
\left|\mathcal{E}_{1}\right|_{\left[-c_{h}-c_{\tau}, t\right]} \leq\left|\mathcal{E}_{1}\right|_{\left[-c_{h}-c_{\tau}, 0\right]}+c_{a} \int_{0}^{t}\left|\mathcal{E}_{1}\right|_{\left[-c_{h}-c_{\tau}, s\right]} \mathrm{d} s .
$$

Hence, Gronwall's inequality (applied to the function $\left|\mathcal{E}_{1}\right|_{\left[-c_{h}-c_{\tau}, t\right]}$ of $t$ ) gives $\left|\mathcal{E}_{1}(t)\right| \leq\left|\mathcal{E}_{1}\right|_{\left[-c_{h}-c_{\tau}, t\right]} \leq$ $|\mathcal{E}|_{\left[-c_{\tau}-c_{h}, 0\right]} e^{c_{a} t}$ for all $t \geq 0$. Since the $\mathcal{E}_{i}$ subsystem in (16) depends affinely on $\mathcal{E}_{i-1}\left(\theta_{i-1}^{-1}\left(\phi\left(\theta_{i}(t)\right)\right)\right)$ and since $\max \left\{\theta_{i-1}^{-1}\left(\phi\left(\theta_{i}(t)\right)\right): 1 \leq i \leq m, t \in[0, \bar{s}]\right\}$ is finite, we can repeat this process $m-1$ times to find a constant $c_{*}>0$ such that

$$
|\mathcal{E}(t)| \leq c_{*}|\mathcal{E}|_{\left[-c_{\tau}-c_{h}, 0\right]}
$$

holds for all $t \in\left[0, s_{*}\right]$, where $s_{*} \geq \bar{s}$ is any upper bound for the arguments of the $\mathcal{E}_{i}$ 's in (17) over all $t \in[0, \bar{s}]$.

Hence, (17) and (52) provide a constant $c_{* *}>0$ such that

$$
|x(t)| \leq c_{* *}|(x, \mathcal{E})|_{\left[-c_{h}-c_{\tau}, 0\right]}
$$

for all $t \in\left[0, s_{*}\right]$, again using Gronwall's inequality. Combining (52) and (53) provides a constant $c_{n}>0$ such that

$$
|(x, \mathcal{E})(t)| \leq c_{n}|(x, \mathcal{E})|_{\left[-c_{h}-c_{\tau}, 0\right]} e^{s_{*}-t}
$$

for all $t \in\left[0, s_{*}\right]$. Similar reasoning gives

$$
\left|\mathcal{E}^{\sharp \sharp}\right|_{\left[a_{*}-2 c_{m}^{\sharp}, a_{*}\right]} \leq c_{* *}|(x, \mathcal{E})|_{\left[-c_{\tau}-c_{h}, 0\right]}
$$

for a large enough constant $c_{* *}>0$. The final uniform globally exponential stability estimate (10) now follows by using (55) and (53) to upper bound the supremum on the right side of (50) and then adding the estimates (50) and (54). This completes the proof of Theorem 1.

\section{ILLUSTRATIONS}

Theorem 1 applies to a broad class of time-varying linear systems for many choices of $h$, including cases where current values $x(t)$ of the original plant state are not available to use in the sequential predictors at times $t$. For drift free systems (where $A=0$ ), our conditions allow any constant measurement delay $\tau \in[0,1 / 2)$, but we can also allow systems with drift. For example, we next illustrate Theorem 1 by revisiting a benchmark example from [12] where we show how Theorem 1 allows a smaller number of predictors than [12] when $\tau=0$. We also explain how Theorem 1 applies in cases where in addition to feedback delays, the measurement delays $\tau(t)$ are nonzero, which were beyond the scope of [12].

The simple pendulum model from [12] is

$$
\left\{\begin{array}{l}
\dot{r}_{1}(t)=r_{2}(t) \\
\dot{r}_{2}(t)=-\frac{g}{l} \sin \left(r_{1}(t)\right)+\frac{1}{\bar{m} l^{2}} v(t-h(t))
\end{array}\right.
$$

where $h(t)$ is the time varying delay in the input $v, l$ is the pendulum length in meters, $\bar{m}$ is the pendulum mass, and $g=9.8 \mathrm{~m} / \mathrm{s}$ is the gravity constant. The objective in [12] was to track a given $C^{1}$ reference trajectory $\left(r_{1, s}(t), r_{2, s}(t)\right)$ that satisfies $\dot{r}_{1, s}(t)=r_{2, s}(t)$. Using the change of feedback

$$
\begin{aligned}
u(t-h(t))= & \frac{1}{\sigma}\left[\frac{1}{\bar{m} l^{2}} v(t-h(t))\right. \\
& \left.-\dot{r}_{2, s}(t)-\frac{g}{l} \sin \left(r_{1, s}(t)\right)\right]
\end{aligned}
$$

where $\sigma>0$ is any constant, we obtain the tracking system

$$
\left\{\begin{aligned}
\dot{\tilde{r}}_{1}(t)= & \sigma \tilde{r}_{2}(t) \\
\dot{\tilde{r}}_{2}(t)= & \frac{g}{\sigma l}\left[\sin \left(r_{1, s}(t)\right)-\sin \left(\tilde{r}_{1}(t)+r_{1, s}(t)\right)\right] \\
& +u(t-h(t)) .
\end{aligned}\right.
$$

for the error variables $\tilde{r}_{1}=r_{1}-r_{1, s}(t)$ and $\tilde{r}_{2}=\left(r_{2}-\right.$ $\left.r_{2, s}(t)\right) / \sigma$.

Our work [14] showed that under the constant delay $h=1$ and with the choices $\sigma=1$ and $r_{1, s}(t)=\omega t$ where $\omega>0$ is a large enough constant, the linearization

$$
\left\{\begin{array}{l}
\dot{x}_{1}(t)=\sigma x_{2}(t) \\
\dot{x}_{2}(t)=-\frac{g}{\sigma l} \cos (\omega t) x_{1}(t)+u(t-h)
\end{array}\right.
$$

of (58) around 0 , in closed loop with the distributed control

$$
\begin{aligned}
u(t)= & -0.6 x_{1}(t)-0.4 x_{2}(t) \\
& -\int_{t-1}^{t}(0.6(t-r-1)+0.4) u(r) \mathrm{d} r,
\end{aligned}
$$

is globally exponentially stable to 0 , and [13] provides a globally asymptotically stabilizing sequential predictor control for the original nonlinear dynamics (58) when $h$ is constant. However, [13] did not apply under time-varying delays.

In [12], we built sequential predictors for the linearized error dynamics (59) with time-varying delays $h$ when $\sigma=1$ 
and $\tau=0$, and the assumptions in [12] were the same as Assumptions 1-2 above in the special case where $\tau=0$. For any constant $\omega>0$, the assumptions from [12] hold using

$$
\begin{aligned}
& A(t)=\left[\begin{array}{cc}
0 & \sigma \\
-\frac{g}{\sigma l} \cos (\omega t) & 0
\end{array}\right], B=\left[\begin{array}{l}
0 \\
1
\end{array}\right], \\
& \text { and } K(t)=\left[\frac{g}{\sigma l} \cos (\omega t)-\sigma \quad-\sigma\right]
\end{aligned}
$$

which gives

$$
|A|_{\infty}=\max \left\{\frac{g}{\sigma l}, \sigma\right\} .
$$

With the preceding choices, and with

$$
h(t)=1+\alpha \sin (t),
$$

and $\sigma=1$, condition (13) above (from [12]) requires that

$$
\begin{aligned}
& \frac{m}{4}-\frac{1+\alpha}{1-\alpha}\left[\frac{|A|_{\infty}}{\sqrt{2}}\left(1+|A|_{\infty}\left(1+\frac{u_{c}}{m}\right)^{m}\right)\left(1+\frac{u_{c}}{m}\right)^{m}\right. \\
& \left.+\left(1+|A|_{\infty}\left(1+\frac{u_{c}}{m}\right)^{m}\right)^{2}\left(1+\frac{u_{c}}{m}\right)\right]>0 .
\end{aligned}
$$

In the special case where $\alpha=1 / 7$ and $\sigma=1$, and for any $\omega>0$ and any $l>g$, the smallest number $m$ of sequential predictors for which (64) holds is $m=47$. On the other hand, for the special case where the measurement delay $\tau$ is 0 and with the preceding choices of the parameters with $\sigma=1$, our requirement (6) is satisfied with $m=17$. Therefore, in the special case of (59) with $\sigma=1$, our new method from Theorem 1 provided a $63 \%$ reduction in the number $m$ of required sequential predictors as compared with [12].

To see how we can also cover cases of nonzero measurement delays $\tau$ in the sequential predictors for (59), first note that in the special case where $\tau$ is constant and with the preceding choices of the parameters with $l>g / \sigma^{2}$, our requirement (12) on the allowable measurement delays $\tau$ is

$$
\tau \in\left[0, \frac{1}{2\left(1+e^{u_{c}} \sigma\right)^{2}}\right),
$$

since $g /(\sigma l) \leq \sigma$, which gives $|A|_{\infty}=\sigma$. Therefore, since $u_{c}$ is independent of $A$, we can allow any constant transmission delay $\tau \in[0,0.5)$ by choosing the number $m$ of sequential predictors to be large enough and $\sigma>0$ small enough. For instance, with the choices $\tau=0.15$, $h(t)=1+\alpha \sin (t)$ with $\alpha=1 / 7$, and $\sigma=0.1$, the smallest integer $m \geq 2$ such that our condition (6) on the number $m$ of required sequential predictors is satisfied is $m=7$.

\section{CONCLUSIONS}

We advanced the theory of sequential predictors for linear time-varying systems with time-varying delays by reducing the number of required sequential predictors as compared with the existing literature, which can reduce the computational burden from applying the method. Our result also incorporates measurement delays that model more realistic cases where the current state of the physical system may not be available for use in the sequential predictors. Such measurement delays had not been considered in previous treatments of sequential predictors for time-varying linear systems with time-varying delays. We hope to develop analogous results for PDE systems with feedback and measurement delays.

\section{REFERENCES}

[1] T. Ahmed-Ali, E. Cherrier, and F. Lamnabhi-Lagarrigue. Cascade high gain predictors for a class of nonlinear systems. IEEE Transactions on Automatic Control, 57(1):221-226, 2012.

[2] T. Ahmed-Ali, I. Karafyllis, and F. Lamnabhi-Lagarrigue. Global exponential sampled-data observers for nonlinear systems with delayed measurements. Systems and Control Letters, 67(7):539-549, 2013.

[3] Z. Artstein. Linear systems with delayed controls: a reduction. IEEE Transactions on Automatic Control, 27(4):869-879, 1982.

[4] N. Bekiaris-Liberis and M. Krstic. Nonlinear Control Under Nonconstant Delays. SIAM, Philadelphia, PA, 2013.

[5] F. Cacace, F. Conte, and A. Germani. Memoryless approach to the LQ and LQG problems with variable input delay. IEEE Transactions on Automatic Control, 61(1):216-221, 2016.

[6] F. Cacace, F. Conte, A. Germani, and G. Palombo. Optimal control of linear systems with large and variable input delays. Systems and Control Letters, 89:1-7, 2016.

[7] F. Cacace, A. Germani, and C. Manes. A chain observer for nonlinear systems with multiple time-varying measurement delays. SIAM Journal on Control and Optimization, 52(3):1862-1885, 2014.

[8] H. Ito, Z-P. Jiang, and P. Pepe. Construction of Lyapunov-Krasovskii functionals for networks of iISS retarded systems in small-gain formulation. Automatica, 49(11):3246-3257, 2013.

[9] H. Khalil. Nonlinear Systems, Third Edition. Prentice Hall, Upper Saddle River, NJ, 2002.

[10] M. Krstic. Delay Compensation for Nonlinear, Adaptive, and PDE Systems. Birkhauser, Boston, 2009.

[11] Mathematica. The world's definitive system for modern technical computing, 2015. http://www.wolfram.com/mathematica/, accessed February 12, 2017.

[12] F. Mazenc and M. Malisoff. New prediction approach for stabilizing time-varying systems under time-varying input delay. In Proceedings of the 55th IEEE Conference on Decision and Control, pages 31783182, Las Vegas, NV, 2016.

[13] F. Mazenc and M. Malisoff. Stabilization of nonlinear time-varying systems through a new prediction based approach. IEEE Transactions on Automatic Control, 62(6):2908-2915, 2017.

[14] F. Mazenc, M. Malisoff, and S-I. Niculescu. Reduction model approach for linear time-varying systems with delays. IEEE Transactions on Automatic Control, 59(8):2068-2082, 2014.

[15] M. Merad, R. Downey, S. Obuz, and W. Dixon. Isometric torque control for neuromuscular electrical stimulation with time-varying input delay. IEEE Transactions on Control Systems Technology, 24(3):971-978, 2016.

[16] W. Michiels and S-I. Niculescu. Stability and Stabilization of TimeDelay Systems. SIAM, Philadelphia, PA, 2007.

[17] S. Mukhopadhyay, C. Wang, M. Patterson, M. Malisoff, and F. Zhang. Collaborative autonomous surveys in marine environments affected by oil spills. In A. Koubaa and A. Khelil, editors, Cooperative Robots and Sensor Networks 2014, pages 87-113. Springer, New York, NY, 2014.

[18] M. Najafi, S. Hosseinnia, F. Sheikholeslam, and M. Karimadin. Closed-loop control of dead time systems via sequential subpredictors. International Journal of Control, 86(4):599-609, 2013.

[19] P. Pepe. On stability preservation under sampling and approximation of feedbacks for retarded systems. SIAM Journal on Control and Optimization, 54(4):1895-1918, 2016.

[20] P. Pepe. Stabilization of retarded systems of neutral type by control Lyapunov-Krasovskii functionals. Systems and Control Letters, 94(2):142-151, 2016.

[21] J-P. Richard. Time-delay systems: an overview of some recent advances and open problems. Automatica, 39(10):1667-1694, 2003.

[22] N. Sharma, C. Gregory, and W. Dixon. Predictor-based compensation for electromechanical delay during neuromuscular electrical stimulation. IEEE Transactions on Neural Systems and Rehabilitation Engineering, 19(6):601-611, 2011.

[23] O. Smith. A controller to overcome dead time. ISA-Journal, 6(2):2833, 1958.

[24] B. Zhou. Pseudo-predictor feedback stabilization of linear systems with time-varying input delays. Automatica, 50:2861-2871, 2014.

[25] B. Zhou. Truncated Predictor Feedback for Time-Delay Systems. Springer-Verlag, Berlin, Germany, 2014.

[26] B. Zhou, Z. Lin, and G-R. Duan. Truncated predictor feedback for linear systems with long time-varying input delays. Automatica, 48:2387-2399, 2012. 